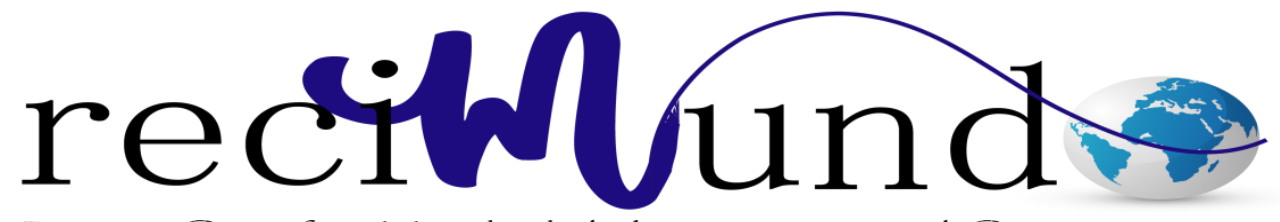

Revista Científica Mundo de la Investigación y el Conocimiento

Estuardo Arafat Escobar Vera ${ }^{\text {a }}$; Luis Alberto Rodríguez Matías ${ }^{\text {b; }}$ Ángela Lastenia Figueroa Córdova ${ }^{c}$; Ángel Bolívar Llerena Hurtado d

Enfermedad de Kienböck: Más de un siglo de conocida. ¿En dónde estamos?

Revista Científica Mundo de la Investigación y el Conocimiento. Vol. 2 núm., 1, febrero, ISSN: 2588-073X, 2018, pp. 583-894

DOI: 10.26820/recimundo/2.1.2018.583-594

Editorial Saberes del Conocimiento

Recibido: 05/12/2017

Aceptado: 10/02/2018
a. estuardoarafat@hotmail.com
b.1arm1978@outlook.com
c._drafigueroa@hotmail.es
d. angel_llerena7@hotmail.com 


\section{Enfermedad de Kienböck: Más de un siglo de conocida. ¿En dónde estamos? \\ Vol. 2, núm. 1., (2018) \\ Estuardo Arafat Escobar Vera; Luis Alberto Rodríguez Matías; Ángela Lastenia Figueroa Córdova; Ángel Bolívar Llerena Hurtado}

\section{RESUMEN}

La Enfermedad de Kienböck, se describió por primera vez en 1910, como una necrosis avascular del hueso semilunar del carpo. Desde sus orígenes hasta la actualidad no se ha logrado encontrar en origen fisiopatológico de esta condición. En el presente artículo se presenta una revisión detallada del problema, desde su enfoque anatómico hasta sus posibles tratamientos descritos hasta la actualidad. Resultados: existen múltiples técnicas descritas para la búsqueda de una curación para esta enfermedad, algunas muy innovadoras con el uso de prótesis, reemplazo de hueso, revascularización, por sólo nombrar algunas. Recomendaciones: todavía queda mucho por investigar y crear para obtener la técnica idónea que logre aportar al paciente mejoría del dolor, movilidad de la articulación y recuperación de la fuerza. Más de 100 años de historia nos dicen que todavía hay más.

Palabras clave: Enfermedad de Kienböck, lunatomalacia, semilunar. 


\title{
Enfermedad de Kienböck: Más de un siglo de conocida. ¿En dónde estamos?
}

Vol. 2, núm. 1., (2018)

Estuardo Arafat Escobar Vera; Luis Alberto Rodríguez Matías; Ángela Lastenia Figueroa

Córdova; Ángel Bolívar Llerena Hurtado

\begin{abstract}
Kienböck's disease was described for the first time in 1910, as an avascular necrosis of the lunate bone of the carpus. From its origins to the present it has not been possible to find physiopathological origin of this condition. In the present article a detailed review of the problem is presented, from its anatomical approach to its possible treatments described to the present. Results: there are multiple techniques described for the search of a cure for this disease, some very innovative with the use of prosthesis, bone replacement, revascularization, to name a few. Recommendations: there is still much to investigate and create to obtain the ideal technique that achieves to provide the patient with pain improvement, mobility of the joint and recovery of strength. More than 100 years of history tell us that there is still more.
\end{abstract}

Keywords: Kienböck disease, lunatomalacia, semilunar. 


\section{Enfermedad de Kienböck: Más de un siglo de conocida. ¿En dónde estamos? Vol. 2, núm. 1., (2018) \\ Estuardo Arafat Escobar Vera; Luis Alberto Rodríguez Matías; Ángela Lastenia Figueroa Córdova; Ángel Bolívar Llerena Hurtado}

\section{Introducción.}

La enfermedad de Kienbök, conocida también como lunatomalacia, osteocondritis del semilunar, condensación del semilunar y necrosis aséptica del semilunar, es un estado clínico caracterizado por el dolor y disminución de la función articular en grado variable de la muñeca, por causa de alteraciones morfológicas del semilunar, provocadas por un trastorno en el aporte sanguíneo (Basterrechea, 1998).

Fue descrita por primera vez por Peste en 1843, sosteniendo que era una lesión traumática (por fractura). Posteriormente, en 1910, Kienböck describió 20 casos reunidos y 16 propios, atribuyendo la enfermedad a cambios nutricionales post-traumáticos, pero no a fractura aguda o fragmentación debida a un episodio traumático único. (García Mata, 1988) Robert Kienböck publicó su clásico tratado "Lunatomalacia" o necrosis avascular del hueso semilunar y sus consecuencias, en donde afirma que el origen de la patología estaría relacionado con una lesión traumática de los vasos y ligamentos que nutren y se insertan en el semilunar. (Paganini, 2013)

Sin embargo, en la actualidad no existe una veracidad, que indique la real causa de la enfermedad. Debido a que ninguna hipótesis sobre su origen ha sido verazmente respaldada por le evidencia científica, por lo cual, sigue siendo una duda el origen de su fisiopatología.

Es por esto que sólo se plantean factores que predisponen a la aparición de la enfermedad de Kienböck. Dentro de su fisiopatología se describe la necrosis de parte o todo el hueso semilunar, en la mayoría de los casos ocasionado por un acortamiento del cúbito. 


\section{Enfermedad de Kienböck: Más de un siglo de conocida. ¿En dónde estamos?}

Vol. 2, núm. 1., (2018)

Estuardo Arafat Escobar Vera; Luis Alberto Rodríguez Matías; Ángela Lastenia Figueroa

Córdova; Ángel Bolívar Llerena Hurtado

En el presente artículo se realizará una descripción de la historia natural, fisiopatología y posibles soluciones para esta enfermedad poco conocida en el área traumatológica.

\section{Materiales y métodos.}

La investigación es de tipo bibliográfica, en la cual se procede a realizar una lectura exhaustiva del material publicado previamente en donde se describan diferentes puntos de vista sobre la patología, valorando cada descripción, argumento, y realizando propiamente una descripción desde el todo hacia las partes.

El análisis de una enfermedad poco conocida permite leer hipótesis establecidas hasta el momento, descripciones que han ido cambiando con el transcurrir de los años; permitiendo de esta manera conocer diferentes enfoques en el origen planteado y por supuesto apreciar la diversidad de criterios terapéuticos con el objetivo de dar fin a este padecimiento que hasta el momento no tiene una forma de resolución definitiva.

Se procedió a la revisión de material bibliográfico en inglés y en español, publicaciones desde las más antiguas hasta las más recientes para poder evaluar a este padecimiento en la historia, y de esta forma apreciar el enfoque cambiante que ha tenido.

Se inicia con la descripción anatómica del problema, luego las hipótesis planteadas de su posible fisiopatología, clínica manifestada, cuadro de clasificación según grado de severidad y alternativas terapéuticas descritas hasta la actualidad. 


\section{Enfermedad de Kienböck: Más de un siglo de conocida. ¿En dónde estamos? Vol. 2, núm. 1., (2018) \\ Estuardo Arafat Escobar Vera; Luis Alberto Rodríguez Matías; Ángela Lastenia Figueroa Córdova; Ángel Bolívar Llerena Hurtado}

\section{Resultados.}

El carpo está compuesto por 8 huesos dispuestos en dos filas, la fila superior comprende, de lateral a medial los huesos escafoides, semilunar, piramidal y pisiforme. Y la fila inferior comprende, de lateral a medial, los huesos trapecio, trapezoide, grande y ganchoso.

Considerado en su conjunto, el macizo óseo carpiano forma un bloque rectangular cuyo diámetro transversal es mayor que el vertical. Su cara posterior es convexa y responde a los tendones extensores de la palma de la mano y de los dedos. Su cara anterior, fuertemente cóncava, constituye el canal del carpo limitado a cada lado por dos importantes salientes: lateral, con los tubérculos del escafoides y del trapecio; medial, con el pisiforme y el gancho del hueso ganchoso. (Latarjet, 2011)

Los huesos del carpo, son huesos cortos, y están formados por una delgada capa de hueso compacto que rodea un bloque de hueso esponjoso. La unión del cúbito, radio y los huesos del carpo conforman lo que conocemos como articulación de la muñeca.

En la descripción anatómica del hueso semilunar se observa lo siguiente: se articula de manera proximal con la cara carpiana del radio, lateralmente con el escafoides, medialmente con el piramidal, y en sentido distal con el hueso grande y el hueso ganchoso.

La irrigación del hueso semilunar se proporciona a través de las dos caras no articulares que posee este hueso (la anterior y la posterior).

Al analizar la biomecánica del hueso semilunar en la enfermedad de Kinböck, se aprecia como en los estadios iniciales no hay diferencia entre los pacientes sanos y los portadores de la 


\section{Enfermedad de Kienböck: Más de un siglo de conocida. ¿En dónde estamos?}

Vol. 2, núm. 1., (2018)

Estuardo Arafat Escobar Vera; Luis Alberto Rodríguez Matías; Ángela Lastenia Figueroa

Córdova; Ángel Bolívar Llerena Hurtado

enfermedad, sin embargo, se puede apreciar a través del estudio tomográfico el aumento de las tensiones sobre la porción osteocondral del hueso (mediante osteoabsorbometría). (Fontaine, 2015)

En estadios más avanzados el semilunar colapsa o se fragmenta, y el escafoides se vuelve horizontal, las tensiones se reducen en la fosa semilunar y aumentan en la fosa escafoidea. Este cambio de cargas es una consecuencia de la destrucción avanzada de semilunar, pero no es la causa de la enfermedad de Kienböck.

La vascularización externa del hueso semilunar depende de la arteria radial, la arteria interósea anterior, la intercarpiana palmar y la arteria interósea anterior y dorsal. La vascularización intraósea puede ser de tres maneras, por una sola arteria en el $8 \%$ de los casos, por dos arterias sin anastomosis intraósea, en el 7\% de los casos y la más común con abundantes anastomosis intraósea en el $85 \%$ de los casos.

En la histología del semilunar afectado se aprecia, áreas de hueso necrótico, alternantes con hueso neoformado. En las áreas de necrosis, hay lagunas vacías, necrosis grasa y osteoide desaparecido. Todo confinado por una interfaz reactiva (zona de reformado) que consiste en hueso formado, tejido de granulación y neovascularización. (Fontaine, 2015)

Luego de la primera descripción de la Enfermedad de Kiembock, se ha intentado encontrar su origen o etiología, sin embargo, esto no ha sido posible hasta la actualidad. Sólo se conocen factores que predisponen a esta enfermedad, dentro de los cuales se pueden nombrar: la forma del hueso semilunar, la forma distal del radio, la diferencia entre el final del cúbito con respecto al radio (aumenta 3.1 veces la posibilidad de tenerla, pero no es concluyente), la 


\section{Enfermedad de Kienböck: Más de un siglo de conocida. ¿En dónde estamos?}

Vol. 2, núm. 1., (2018)

Estuardo Arafat Escobar Vera; Luis Alberto Rodríguez Matías; Ángela Lastenia Figueroa Córdova; Ángel Bolívar Llerena Hurtado

superficie del hueso semilunar que es cubierta por el radio, insuficientes anastomosis intraóseas, y la excesiva presión intraósea con estasis venoso.

Se sugiere que existen condiciones personales predisponentes como es el caso del sexo femenino, la mano dominante, esfuerzos físicos intensos, y algunas enfermedades asociadas como es el caso de la esclerosis múltiple, artritis reumatoidea, enfermedad de Crohn, Lupus eritematoso sistémico, anticuerpos antifosfolípidos, dermatomiositis entre otras.

Las hipótesis actuales descartan la posibilidad de microtraumas, traumas repetitivos, y factores mecánicos como generadores de esta enfermedad. Sin embargo, todavía no existe una causa comprobada por la cual se genere el proceso microvascular de la necrosis a nivel del semilunar. Se cree que se puede generar por un fenómeno biológico en la cual se genera un desequilibrio entre la resorción osteoclástica y la reparación osteoblástica, probablemente mediado por un mecanismo genético desconocido para el momento. (Fontaine, 2015)

Dentro de la clínica se describe que se ve manifestada en pacientes jóvenes, trabajadores manuales, con molestias generalmente en la región dorsal del carpo, más específicamente en la fosa de crucifixión. En los estadios iniciales se presenta intermitente, o sólo al sobre uso de la articulación. Con el paso del tiempo la articulación va perdiendo motilidad, tornándose rígida con afectación en la fuerza.

Estadios de la Enfermedad de Kienböck según los hallazgos Radiológicos. (Escala de

\section{Lichtman):}

\begin{tabular}{|l|l|}
\hline Estadio I & Normal \\
\hline Estadio II & Incremento de la densidad ósea homogénea o heterogénea. \\
\hline Estadio III-A & Deformidad Semilunar con indentación proximal o radial. Fractura Lineal. \\
\hline
\end{tabular}




\section{Enfermedad de Kienböck: Más de un siglo de conocida. ¿En dónde estamos?}

Vol. 2, núm. 1., (2018)

Estuardo Arafat Escobar Vera; Luis Alberto Rodríguez Matías; Ángela Lastenia Figueroa Córdova; Ángel Bolívar Llerena Hurtado

\begin{tabular}{|l|l|}
\hline & Colapso semilunar. \\
\hline Estadio III-B & Inestabilidad carpiana comprobada \\
\hline Estadio IV & Osteoartritis \\
\hline
\end{tabular}

Muchas técnicas ha sido propuestas para el tratamiento de ésta enfermedad, comenzando por los estadios iniciales (Estadio I y II), donde la inmovilización genera acalmia del dolor, sin embargo, no detiene el proceso degenerativo. Todo acompañado de la prevención de la lesión mediante la no realización de movimientos vibratorios y repetitivos (martillos hidráulicos, taladros, engrapadoras, entre otros), evitar la hiperextensión o el manejo de peso, son todas estrategias que previenen las crisis intermitentes de dolor.

En estadios más avanzados, tipo III-B o IV ya se plantean resoluciones quirúrgicas innovadoras, las cuales se harán mención a continuación, haciendo la acotación de que ninguna técnica a aportado una resolución verdaderamente eficaz.

- Técnica de Graner: artrodesis intercarpiana limitada, con osteotomía del hueso grande, previa lunarectomía, extrayendo injerto de cresta iliaca. En una serie de 1 paciente, se logró detener la evolución de la enfermedad, con una ganancia en cuanto a mejoría del dolor y de la fuera, sin embargo, con pérdida de la motilidad. (García Mata, 1988)

- Osteotomía del Radio sin acortamiento: la operación pretende reducir la sobrecarga axial longitudinal del antebrazo, debida a la fuerza muscular, sobre la mitad radial del semilunar. En una serie realizada en 5 casos, se observó la mejoría franca de la sintomatología, sin aparente modificación de la diferencia existente entre cúbito y radio. Es una serie pequeña, en vías de aprobación. (Blanco., 1997.) 


\section{Enfermedad de Kienböck: Más de un siglo de conocida. ¿En dónde estamos?}

Vol. 2, núm. 1., (2018)

Estuardo Arafat Escobar Vera; Luis Alberto Rodríguez Matías; Ángela Lastenia Figueroa Córdova; Ángel Bolívar Llerena Hurtado

- Denervación Total de la muñeca (Técnica de Wilhelm): en ésta técnica se procede mediante una mínima incisión realizar la denervación de la articulación. Este método no elimina la progresión de la enfermedad, sin embargo, provee un alivio incluso desde el 1er mes posterior a la enfermedad, logrando hacer que el paciente pueda volver a su actividad laboral habitual. Provee calidad de vida, a pesar de no mejorar fuerza, ni motilidad. (Braga-Silva, 2011)

- Reemplazo del semilunar con Tróclea medial femoral vascularizada: esta técnica provee una intervención utilizando hueso esponjoso cuya respuesta a la vascularización es satisfactoria. No mejora la motilidad ni la fuera, pero detiene el proceso. (Simmons, Tobias, \& Lichtman, 2009).

- Prótesis de Silicona: en la cual se extrae el hueso semilunar, se implanta una prótesis de silicona, realizando una estabilización de la articulación con el hemitendón del palmar mayor. Sin embargo, esta técnica a pesar de grande resultados en cuanto a mejoría de la clínica sin pérdida de fuerza y gran movilidad, presenta la complicación de la presencia de sinovitis por el silicón de la prótesis. (Paganini, 2013)

- Descarga Temporal: ésta técnica busca eliminar la carga sobre el semilunar mediante la fijación externa "casting", debe ir acompañada de un proceso de revascularización o injerto de hueso esponjoso. Sin embargo, en las series realizadas con posterior autopsia en cadáveres sólo se ha evidenciado disminución en el dolor, sin apreciar mejoría de la necrosis a vascular. (Bain, 2015).

\section{Conclusiones.}




\section{Enfermedad de Kienböck: Más de un siglo de conocida. ¿En dónde estamos?}

Vol. 2, núm. 1., (2018)

Estuardo Arafat Escobar Vera; Luis Alberto Rodríguez Matías; Ángela Lastenia Figueroa Córdova; Ángel Bolívar Llerena Hurtado

La Enfermedad de Kienböck es una patología con una larga historia de investigación desde su primera descripción en 1910. Sin embargo, ha sido muy frustrante para la ciencia médica en su búsqueda continua para la mejoría de ésta, logrando obtener pequeños avances en el tratamiento de la sintomatología, a través de la vía quirúrgica en estadios avanzados, pero sin lograr conseguir un método que se convierta en la solución definitiva y comprobada de este mal. Se puede concluir que en estadios iniciales, la inmovilización genera acalmia del dolor, y en estadios más avanzados, todavía estamos a la espera de métodos innovadores que logren dar respuesta al padecimiento de tantos pacientes.

Recomendaciones: En vista de los hallazgos descritos anteriormente, se recomienda al lector, el buen análisis de cada caso de paciente con Enfermedad de Kienböck, para buscar la mejor alternativa individualizada, en aras de mejorar la calidad de vida del paciente portador. Sin embargo, esto nos alienta a seguir buscando técnicas que sean desarrolladas para obtener una clara solución mediante el conocimiento de esta enfermedad, de la cual actualmente no se conocen bien sus mecanismos fisiopatológicos. Quizás con los avances que cada día nos muestra la ciencia, se logrará deducir la causa real de ésta enfermedad y de esa manera, desde su origen buscar la posible solución.

\section{Bibliografía.}

Bain, I. (2015). Kienböck Disease: Recent Advances in the Basic Science, Assessment and Treatment. Hand Surgery, 20(3), 352-365.

Basterrechea, F. L. (1998). Enfermedad De Kienböck Bilateral. Rev Cubana Med Milit, 141-5. 


\section{Enfermedad de Kienböck: Más de un siglo de conocida. ¿En dónde estamos?}

Vol. 2, núm. 1., (2018)

Estuardo Arafat Escobar Vera; Luis Alberto Rodríguez Matías; Ángela Lastenia Figueroa Córdova; Ángel Bolívar Llerena Hurtado

Braga-Silva, J. (2011). Wrist denervation for painful conditions of wrist. J. Hand Surg. Am, 36(6), 961-966.

Fontaine, C. (2015). Kienböck’s disease. Chirurgie de la main, 4-17.

García Mata, S. (1988). Tratamiento de la Enfermedad de Kienböck avanada mediante la técnica de Graner. Revista Española de Cirugía Osteoarticular, 23(137), 347-356.

Latarjet, M. (2011). Anatomía Humana. Buenos Aires: Panamericana.

Paganini, F. (2013). Enfermedad de Kienböck tratada con prótesis de hueso semilunar. Reporte de un caso. Rev Asoc Argent Ortop Traumatol, 23(3), 36-42.

Simmons, S., Tobias, B., \& Lichtman, D. (2009). Lunate revascularization with artery implantation and bone grafting. J Hand Surg Am., 34(1), 155e-163e. 